\title{
Dynamic critical properties of a one-dimensional probabilistic cellular automaton
}

\author{
Pratip Bhattacharyya* \\ Low Temperature Physics Section \\ Saha Institute of Nuclear Physics \\ Sector - 1, Block - AF, Bidhannagar \\ Calcutta 700 064, India.
}

\begin{abstract}
Dynamic properties of a one-dimensional probabilistic cellular automaton are studied by monte-carlo simulation near a critical point which marks a second-order phase transition from a active state to a effectively unique absorbing state. Values obtained for the dynamic critical exponents indicate that the transition belongs to the universality class of directed percolation. Finally the model is compared with a previously studied one to show that a difference in the nature of the absorbing states places them in different universality classes.
\end{abstract}

*E-mail : pratip@hp1.saha.ernet.in 


\section{Introduction}

Discrete models of nonequilibrium stochastic processes form a class of interacting particle systems [1]. Of the models studied with short range and translationally invariant interactions in space and time, the ones exhibiting a continuous phase transition from an active steady state to an absorbing state fall into two universality classes [2] :

(1) the class of directed percolation (DP),

(2) the class of parity conservation (PC).

Models with a unique absorbing state have been conjectured to belong to the DP class [3, 41. This is yet the larger of the two classes and includes, for example, lattice models of directed percolation in $d+1$ dimensions [5], the contact process for an epidemic [6], Schlögl's first and second models of autocatalytic reactions [7], the Domany-Kinzel automaton [8, 9], a lattice version of reggeon field theory [10] and branching annihilating random walks with an odd number of offsprings [11]. The order parameter is usually the density of 'particles' (occupied sites, kinks). The models mentioned above are one-component systems and therefore

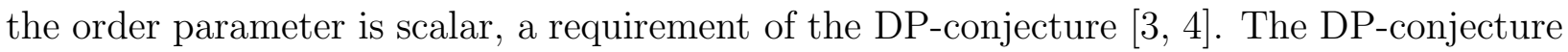
was generalised to include multicomponent systems such as the ZGB model of heterogeneous catalysis [12, 13, 14] and interacting dimer-trimer models [15]. The class of DP was shown to further include systems with an infinite number of absorbing states [16]; these absorbing states are frozen configurations characterised by unique statistical properties, a lack of long range correlations and a general lack of symmetry among them.

On the other hand, models with degenerate, mutually symmetric absorbing states are believed to belong to the parity-conserving class where the number of 'particles' are conserved modulo 2. Prominent examples are the probabilistic cellular automaton models $A$ and $B$ of [17, 18], an interacting monomer- dimer model [19] and branching annihilating random walks (BAW) with an even number of offsprings [20]. According to one point of view [2, 18] the mechanism that puts these models in a class different from that of DP is the conservation of particle number modulo 2. This point was proved for BAW with an even number of offsprings: introduction of spontaneous annihilation of particles in the model destroyed the conservation of their number modulo 2 and the critical behaviour of this modified model was observed to be in the class of DP [21]. According to a second point of view the critical behaviour of the models in the PC class is due to the symmetry among its absorbing states [22, 23]. To prove the point the interacting monomer-dimer model of [19, in the presence of 
a weak parity-conserving field that destroyed the symmetry among the absorbing states, was shown to exhibit critical behaviour in the DP class [22]. This view was further emphasized in the generalised versions of the Domany-Kinzel automaton and the contact process [23]. In these generalised models there was no explicit parity-conservation law and with two symmetric absorbing states the critical behaviour was in the PC class. In the presence of a symmetry breaking field the critical behaviour of these models changed to the DP class. However in the case of the probabilistic cellular automata of [17, 18 both the conservation of particle number modulo 2 and mutual symmetry among the absorbing states are present and therefore it it is not apparent which of these two features is responsible for the models to be in the PC class.

In this paper I shall study, using time-dependent monte-carlo simulations, the dynamic critical properties of a one-dimensional probabilistic cellular automaton [25] which has three absorbing states and exhibits a phase transition from a active state to one of them only. The simulations provide values for the critical point (more accurate than previous estimates 25 , 26]) and the dynamic critical exponents that decide the universality class to which the phase transition belongs.

\section{The model}

The probabilistic cellular automaton studied here is 'elementary' (in the sense of Wolfram [24]) with two states per site and translationally invariant nearest neighbour interactions. The probabilistic behaviour enters the model through two mutually symmetric components of the evolution rule (like specific noise added to a otherwise deterministic system), the rest of the rule components being deterministic in nature.

Formally the model [25] is defined as a line of sites with a binary variable $x_{i} \in\{0,1\}$ assigned to each site $i$. A site is said to be occupied if $x_{i}=1$ and unoccupied otherwise. Starting from a given configuration $\left\{x_{i}^{(0)}\right\}$ the system evolves by parallel update of the variable $x_{i}$ at all lattice sites following a local rule of evolution. With nearest neighbour interactions the evolution rule is defined by a set of eight components $\left[x_{i-1}^{(t)}, x_{i}^{(t)}, x_{i+1}^{(t)}\right] \mapsto$ $x_{i}^{(t+1)}$ corresponding to $2^{3}$ distinct three-site neighbourhoods : 


$$
\begin{aligned}
& \frac{t:}{t+1:} \quad \frac{111}{0} \quad \frac{110}{0} \quad \frac{011}{0} \quad \frac{101}{0} \quad \frac{010}{1} \quad \underbrace{\underline{100} \quad \underline{001}} \quad \frac{000}{0} \\
& 1 \text { with probability } p \\
& 0 \text { with probability } 1-p
\end{aligned}
$$

The evolution rule (1) thus follows, according to Wolfram's nomenclature scheme [24], 'elementary' rule 4 with probability $1-p$ and 'elementary' rule 22 with probability $p$.

Clearly, the components of the evolution rule are of two kinds: (1) active components, where the central site changes its value, and (2) passive components, where the value of the central site remains unchanged. The dynamic evolution of the system is due to the active components. This involves two opposing processes :

(a) Annihilation of adjacent occupied sites (multi-'particle' annihilation) due to the rule components $111 \mapsto 0,110 \mapsto 0,011 \mapsto 0$ prevents the survival of occupied pairs of nearest neighbours; this is a deterministic process.

(b) Creation of an occupied site $(100 \mapsto 1,001 \mapsto 1)$ requires an unoccupied site to have exactly one occupied neighbour; this process of creation occurs only with a probability $p$. The multi-'particle' annihilation and creation of 'particles' can, in effect, give rise to a diffusion process - if a 'particle' at site $i$ gets annihilated after creating another 'particle' at a neighbouring site $i+r$, it has effectively taken a step from $i$ to $i+r$.

The passive components determine the absorbing states of the model. Since there is no spontaneous creation of occupied sites $(000 \mapsto 0)$ the 'vacuum' (all sites unoccupied) is always an absorbing state :

$$
\text { Absorbing state I : } \quad x_{i}=0 \text { for all } i .
$$

Again, it is evident from the rule components $010 \mapsto 1$ and $101 \mapsto 0$ that an occupied site with unoccupied neighbours remains occupied and vice versa. These features of the evolution rule lead to two mutually symmetric absorbing states :

$$
\begin{array}{ll}
\text { Absorbing State II : } & x_{i}= \begin{cases}0 & \text { for } i=\text { even, } \\
1 & \text { for } i=\text { odd. }\end{cases} \\
\text { Absorbing State III : } & x_{i}= \begin{cases}1 & \text { for } i=\text { even, } \\
0 & \text { for } i=\text { odd } .\end{cases}
\end{array}
$$


The main point of interest in this paper is a phase transition exhibited by the model [25]. For $p$ less than a critical value $p_{c}$ there exists three distinct steady states given by the three absorbing states of the model. For all initial states but two, it has been observed in computer simulations [25] that the only steady state is the 'vacuum' (absorbing state I). The two cases of exception occur when the initial state is either absorbing state II or absorbing state III, which must also be respectively the steady states of the system. Evolving from any other initial state the mutually symmetric absorbing states II and III are never reached. This can be understood by the fact that these states are fixed points that repel - a damage introduced in these two states by flipping only a single bit spreads through the entire lattice and eventually the 'vacuum' is reached. Above $p_{c}$ there is another steady state called the 'active' state with a constant non-zero density of occupied sites. The active state is stable only on an infinite lattice; for finite lattices it occurs as a metastable state that will decay to the 'vacuum' if allowed to evole for sufficiently long time. On an infinite lattice, in the supercritical region $\left(p>p_{c}\right)$, all possible initial states other than the three absorbing states evolve into the 'active' steady state. The density of occupied sites $\rho_{\infty}$ in the steady state acts as the order parameter for this phase transition - in the supercritical region $\rho_{\infty}$ goes to zero continuously as $p$ approaches $p_{c}$ [25:

$$
\rho_{\infty} \sim\left(p-p_{c}\right)^{\beta}, \quad p \rightarrow p_{c+},
$$

where $\beta$ is the critical exponent for the order parameter. For random initial states the model therefore makes a continuous (second order) phase transition, at $p=p_{c}$, from a active state with $\rho_{\infty}>0$ to a effectively unique absorbing state, the 'vacuum'. Because of the conjecture of [3, 4] this phase transition is expected to belong to the universality class of DP.

\section{Dynamic properties at the critical point}

The phase transition is characterised here by critical exponents describing the dynamic properties of the model at the point of transition (the critical point $p_{c}$ ). To do so dynamic properties of the model are studied by monte-carlo simulations on a computer, only close to $p_{c}$. While the study of steady state properties require simulations starting from disordered states, the dynamic properties are studied using initial states with a single occupied site. Following the evolution rule (1) an initial occupied site grows into a cluster; the position of this initial occupied site is called the 'origin' of the cluster. For each value of $p, 10^{4}$ clusters 
were simulated. Each cluster was allowed to evolve for 5000 time steps, unless it had died out earlier. Typical examples of evolution near the critical point are shown in figure 1.

The quantities measured are : (1) the survival probability $P(t)$, which gives the chance that there is at least one occupied site after $t$ time steps, (2) the average number of occupied sites $N(t)$ after $t$ time steps, and (3) the mean square radius $R^{2}(t)$ of the cluster (or, the mean square displacement from the 'origin' of the cluster) after $t$ time steps. At the critical point $p=p_{c}$, these quantities are expected to follow power-type scaling laws in the long-time $\operatorname{limit}(t \rightarrow \infty)$ :

$$
\begin{aligned}
P(t) & \sim t^{-\delta} \\
N(t) & \sim t^{\eta} \\
R^{2}(t) & \sim t^{z}
\end{aligned}
$$

where $\delta, \eta$ and $z$ are dynamic critical exponents. In the case of $N(t)$ the average is taken over all clusters including those which have died out, while $R^{2}(t)$ is averaged over the occupied sites in the surviving clusters only.

Results for the three quantities $P(t), N(t)$ and $R^{2}(t)$, obtained from computer simulations of the model (1), are shown in figure 2. On log-log plot curves in the subcritical region bend downward while those in the supercritical region bend upward; at the critical point the curves are expected, if the scaling laws (6) are true, to be straight lines as $t \rightarrow \infty$. It is obvious from figure 2 that $0.75<p_{c}<0.753$. More precise estimates for $p_{c}$ and the critical exponents are obtained by the method of effective exponents due to Grassberger [27. Effective exponents $\delta_{t}, \eta_{t}$ and $z_{t}$ are defined as the local slopes of the curves shown in figure 2 :

$$
-\delta_{t}=\frac{\Delta[\log P(t)]}{\Delta[\log t]}, \quad \eta_{t}=\frac{\Delta[\log N(t)]}{\Delta[\log t]}, \quad z_{t}=\frac{\Delta\left[\log R^{2}(t)\right]}{\Delta[\log t]},
$$

which are measured by using the formulae [27] :

$$
-\delta_{t}=\frac{\log [P(t) / P(t / m)]}{\log m}
$$

and similar expressions for $\eta_{t}$ and $z_{t}$. Results for $m=10$ are shown in figure 3 . Like the curves in figure 2, curves for $p<p_{c}$ bend downward while those for $p>p_{c}$ bend upward.

For finite times the dynamic quantities do not have a pure power-law scaling form like (6); in general there exists correction-to-scaling of the type : 


$$
P(t) \sim t^{-\delta}\left(1+\frac{a}{t}+\frac{b}{t^{\delta^{\prime}}}+\cdots\right)
$$

and similar expressions for $N(t)$ and $R^{2}(t)$ with correction-to-scaling exponents $\delta^{\prime}, \eta^{\prime}$ and $z^{\prime}$ respectively. Consequently the behaviour of the effective exponents defined in (7) are given by [27] :

$$
\delta_{t}=\delta+\frac{a}{t}+\frac{\delta^{\prime} b}{t^{\delta^{\prime}}}+\cdots
$$

and similar expressions for $\eta_{t}$ and $z_{t}$. The critical exponents of the model appear as the asymptotic values of the corresponding effective exponents :

$$
\delta=\lim _{t \rightarrow \infty} \delta_{t}, \quad \eta=\lim _{t \rightarrow \infty} \eta_{t}, \quad z=\lim _{t \rightarrow \infty} z_{t} .
$$

Therefore in a plot of an effective exponent (as the ordinate) versus $1 / t$ (as the abscissa) the corresponding critical exponent is obtained as the intercept of the curve for $p=p_{c}$ on the ordinate axis. Using this method the following estimates for the critical characteristics of the model were obtained from computer simulation data :

$$
p_{c}=0.7515 \pm 0.0005
$$

and

$$
\begin{aligned}
\delta & =0.16 \pm 0.01, \\
\eta & =0.32 \pm 0.02, \\
z & =1.27 \pm 0.01 .
\end{aligned}
$$

The value of the critical point agrees closely with previous estimates [25, 26] and improves upon them. The values of the critical exponents are also found to satisfy the scaling relation 110 :

$$
d z=2 \eta+4 \delta
$$

where $d$ is the number of spatial dimensions of the system (here $d=1$ ).

Evolving from disordered initial states, the density of occupied sites $\rho_{t}$ was observed to follow the same dynamic scaling as the survival probability. In computer simulations of 25] 
the exponent characterising the critically slow relaxation $\rho_{t} \sim t^{-\alpha}$ at $p=p_{c}$ was found to be $\alpha \approx 0.16 \approx \delta$.

The values of all the three dynamic critical exponents agree, within the limits of error, with the corresponding values for DP in $1+1$ dimensions [10, 28, 29]. In a previous work [26] the critical exponents $\nu_{\perp}$ and $\nu_{\|}$for the correlation length and correlation time respectively at this particular phase transition, obtained by finite-size scaling methods, were also found to belong to the DP class. However, the order parameter exponent $\beta$ was observed to disagree with the DP value [25]; this was an error arising out of finite-size effects and fluctuations due to the small sample size used for averaging. Since $\delta$ and $\nu_{\|}$are already in the class of DP, the exponent $\beta$, by virtue of the relation $\beta=\delta \nu_{\|}$[10], must also agree with the directed percolation value for $1+1$ dimensions.

The last result is concerned with the fractal dimension of the clusters in the single space dimension at $p=p_{c}$. The average number of occupied sites per surviving cluster is given by $N_{s}(t)=N(t) / P(t)$. The fractal dimension $d_{F}$ of the clusters at fixed time is defined by :

$$
N_{s} \sim R^{d_{F}}
$$

Following the definition of the dynamic exponents (6) the fractal dimension is expected to satisfy the relation :

$$
d_{F} z=2(\eta+\delta) \text {. }
$$

Figure 4 shows a $\log -\log$ plot of $N_{s}$ versus $R$ at $p=p_{c}$. The slope of the curve is given by :

$$
d_{F}=0.74 \pm 0.02
$$

which satisfies relation (16) within the limits of error.

\section{Discussion}

In this concluding section I shall compare the model defined by (11) with another that has the same set of absorbing states and that belongs to a different university class.

The probabilistic cellular automaton (11) studied here was found to have three absorbing states given by (2), (3) and (4). For $p<p_{c}$ the 'vacuum' (absorbing state I) is the only attractor of the model while the other two absorbing states (II and III) are never reached 
from disordered initial states. Consequently the 'vacuum' appears, in effect, to be the unique absorbing state in the subcritical region. The dynamic critical exponents characterising the phase transition in this model indicate that the transition belongs to the class of DP, in agreement with the conjecture of [3, 团.

On the other hand, the phase transition occuring in model $A$ of [17 belongs to the PC class. This model is yet another one-dimensional elementary probabilistic cellular automaton defined by the evolution rule :

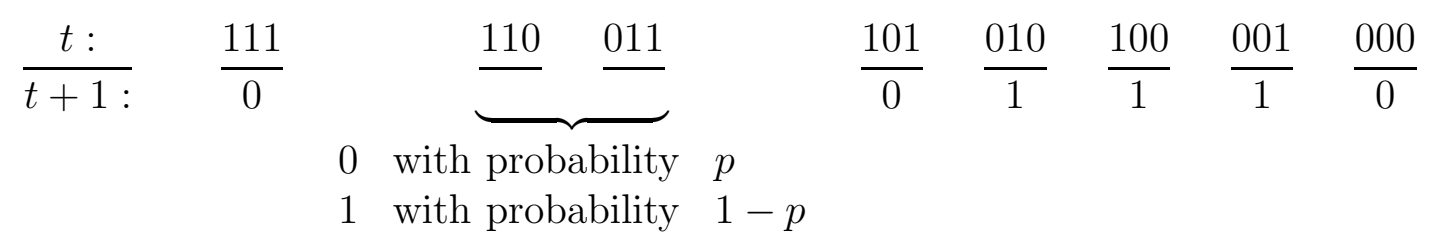

It is remarkable that the three absorbing states of this model are exactly the same as those of model (1). However, contrary to their nature in model (11), the mutually symmetric absorbing states II and III occur as attractors of this model for $p<p_{c}$ while aborbing state I (the 'vacuum') is never reached from disordered initial states. It appears that the contrast in the nature of the absorbing states between the two models places them in different universality classes. In that case the non-DP behaviour of model (18) must be due to the degeneracy in the absorbing state in the subcritical region, thus supporting the view of [22, 23].

\section{Acknowledgment}

I am grateful to Professor Bikas K. Chakrabarti for discussions and for critically reading the manuscript. The work was supported by CSIR, Government of India. 


\section{References}

[1] Ligget T M 1985 Interacting Particle Systems (New York: Springer-Verlag).

[2] Grassberger P 1995 J. Stat. Phys. 7913.

[3] Janssen H K 1981 Z. Phys. B 42151.

[4] Grassberger P 1982 Z. Phys. B 47365.

[5] Kinzel W 1983 Directed Percolation Percolation Structures and Processes, Annals of Israel Physical Society Vol. 5, ed G Deutscher, R Zallen and J Adler (Bristol: Adam Hilger).

[6] Harris T E 1974 Ann. Prob. 2969.

[7] Schlögl F 1972 Z. Phys. B 253147.

[8] Domany E and Kinzel W 1984 Phys. Rev. Lett. 53311.

[9] Kinzel W 1985 Z. Phys. B 58229.

[10] Grassberger P and de la Torre A 1979 Ann. Phys. 122373.

[11] Jensen I 1993 Phys. Rev. E 47 R1.

[12] Ziff R M, Gulari E and Barshad Y 1986 Phys. Rev. Lett. 562553.

[13] Grinstein G, Lai Z W and Browne D A 1989 Phys. Rev. A 404820.

[14] Jensen I, Folgedby H C and Dickman R 1990 Phys. Rev. A 413411.

[15] Albano E V 1995 Physica A 214426.

[16] Jensen I 1993 Phys. Rev. Lett. 701465.

[17] Grassberger P, Krause F and von der Twer T 1984 J. Phys. A: Math. Gen. 17 L105.

[18] Grassberger P 1989 J. Phys. A: Math. Gen. 22 L1103.

[19] Kim H H and Park H 1994 Phys. Rev. Lett. 732579.

[20] Jensen I 1994 Phys. Rev. E 503623. 
[21] Jensen I 1993 J. Phys. A: Math. Gen. 263921.

[22] Park H and Park H 1995 Physica A 22197.

[23] Hinrichsen H 1997 Phys. Rev. E 55219.

[24] Wolfram S 1983 Rev. Mod. Phys. 55601.

[25] Bhattacharyya P 1996 Physica A 234427.

[26] Bhattacharyya P Physica A (in press).

[27] Grassberger P 1989 J. Phys. A: Math. Gen. 223673.

[28] Essam J W, Guttmann A J and de Bell K 1988 J. Phys. A: Math. Gen. 213815.

[29] Dickman R and Jensen I 1991 Phys. Rev. Lett. 672391. 


\section{Figure Captions}

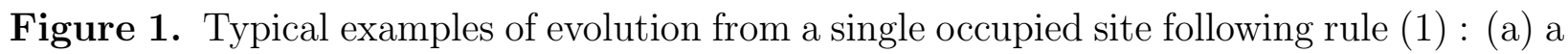
case in the subcritical region $(p=0.74)$ and $(\mathrm{b})$ a case in the supercritical region $(p=0.76)$. The first 200 time-steps if the evolution process are shown.

Figure 2. Results for three dynamic properties from monte-carlo simulations of the model : (a) survival probability, (b) average number of occupied sites and (c) mean square radius of the evolving cluster. Each of the three panels contains five curves that correspond to $p=$ 0.753 (top), 0.752, 0.7515, 0.751, and 0.75 (bottom) respectively.

Figure 3. The effective exponents measured as the local slopes of the curves shown in figure 2. For large $t$, data have been averaged over many time-steps in order to suppress fluctuations.

Figure 4. The log-log plot of the average number of occupied sites as a function of the root-mean-square radius of the clusters at $p=p_{c}$. The slope of the curve gives the fractal dimension of the clusters at fixed time. The straight line drawn below the curve is the graph of $N_{s}=$ const. $R^{0.74}$. 


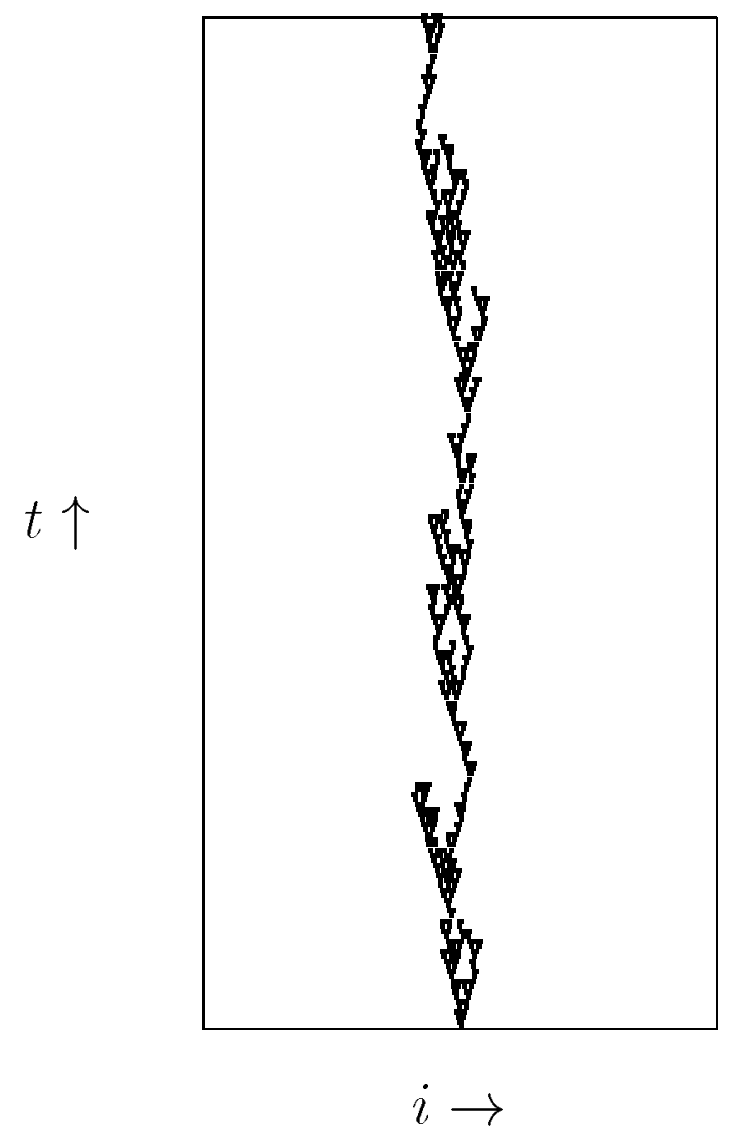

Figure 1(a) 


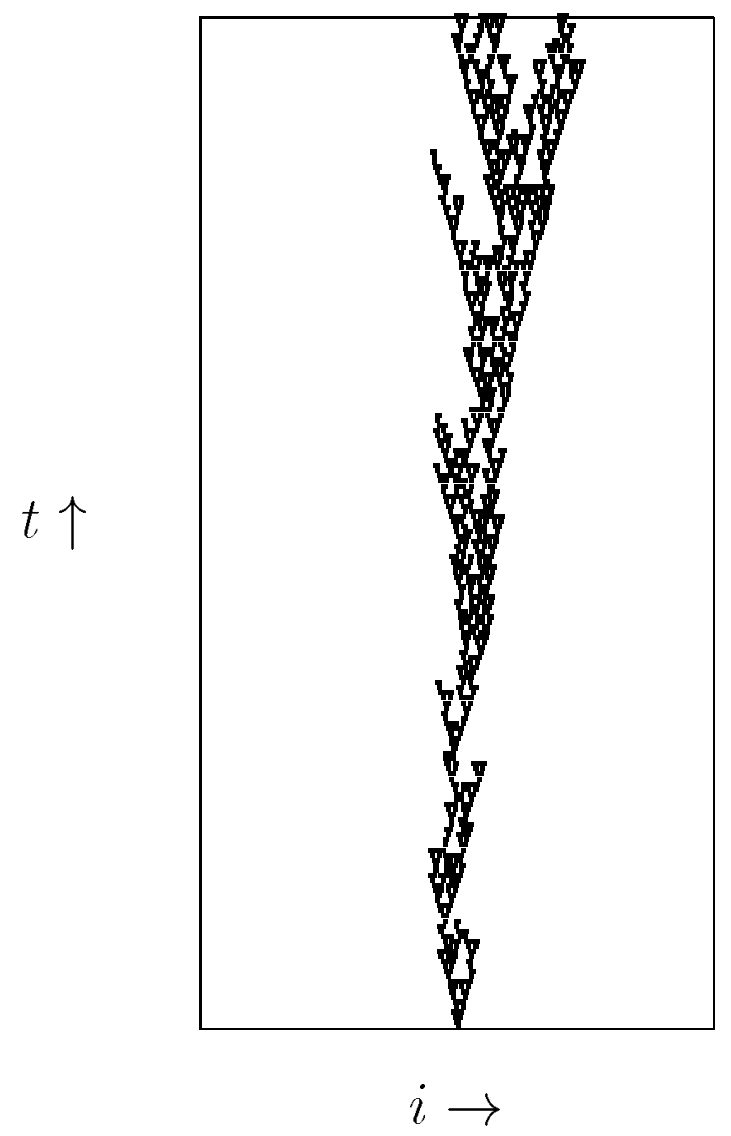

Figure 1(b) 


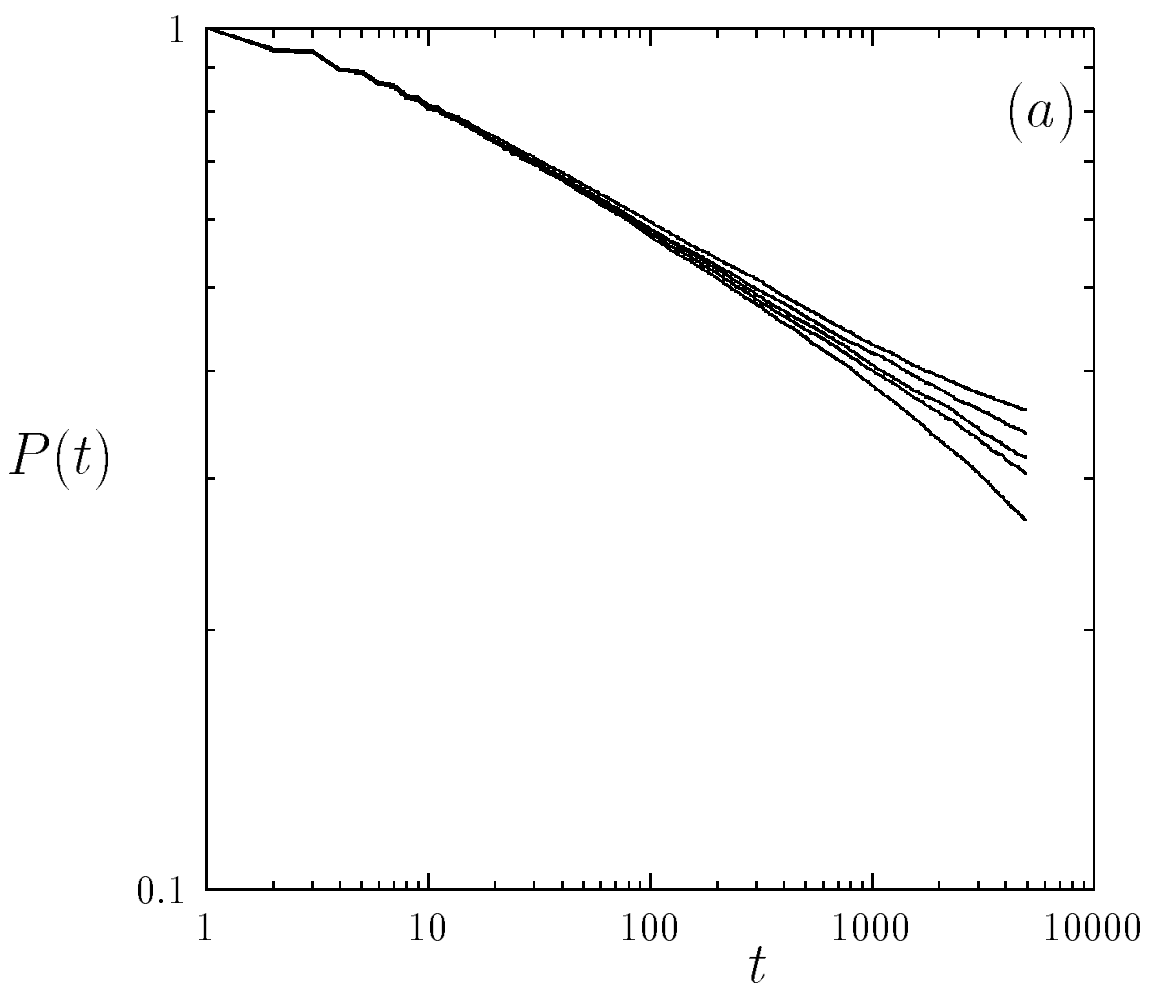

Figure 2(a) 


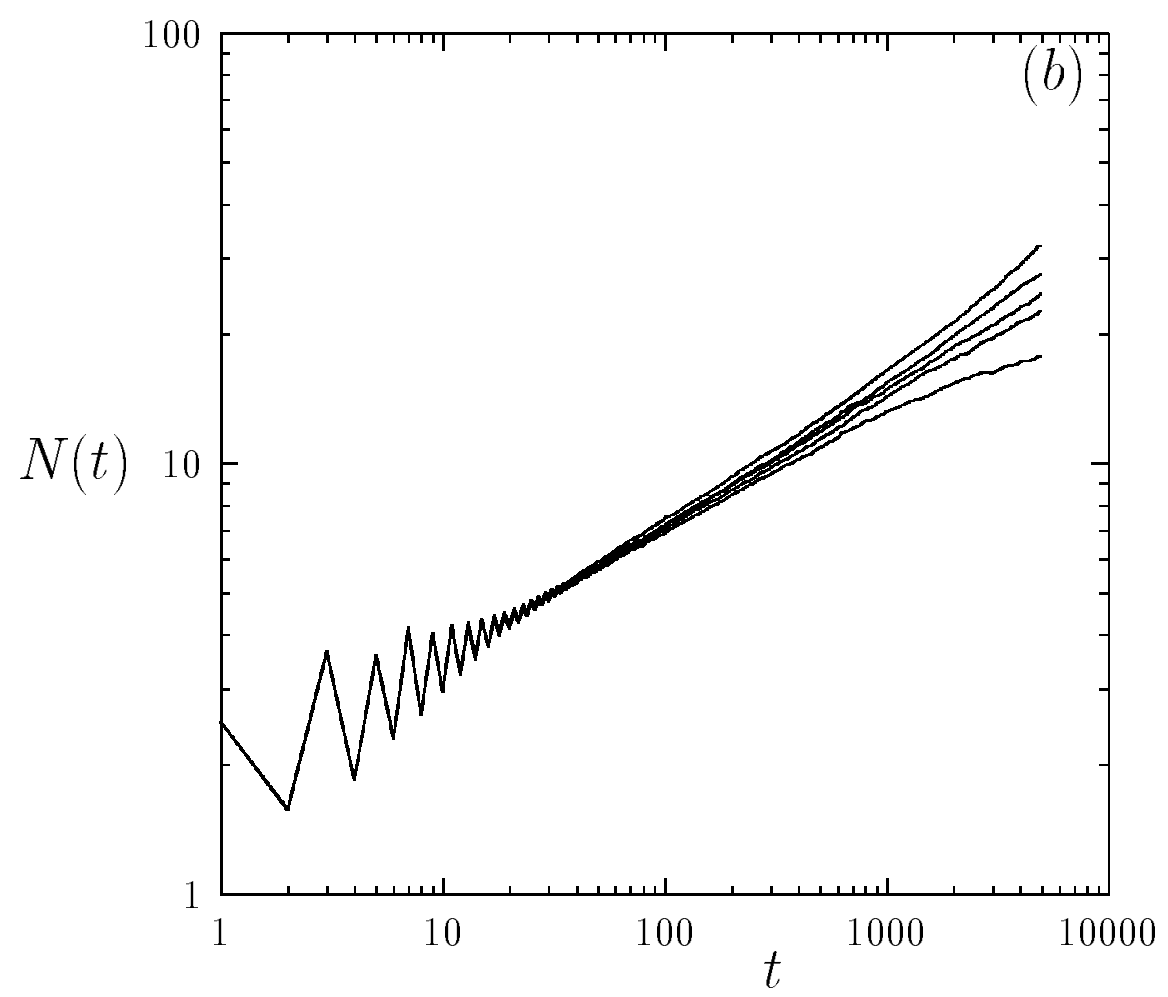

Figure 2(b) 


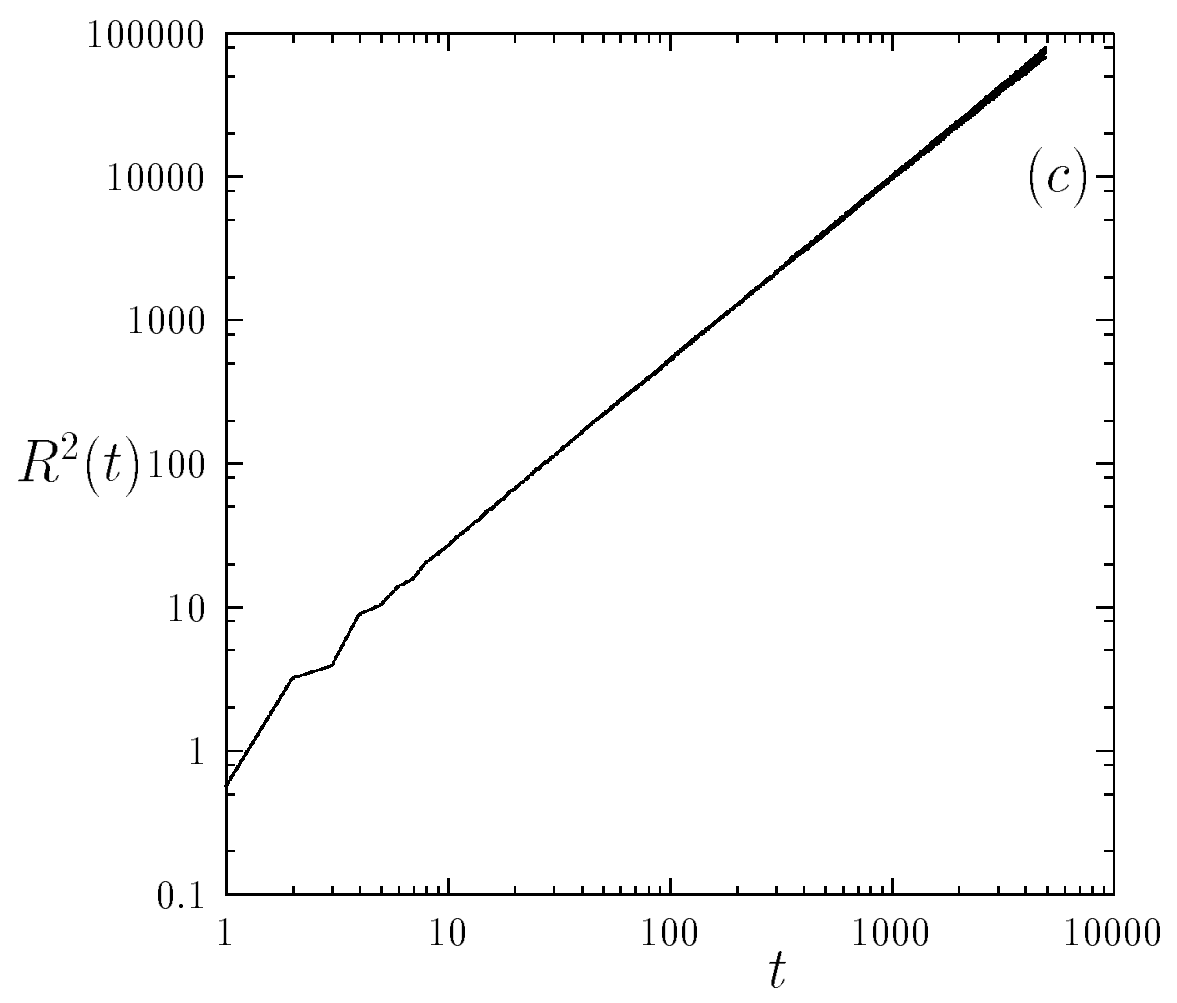

Figure 2(c) 


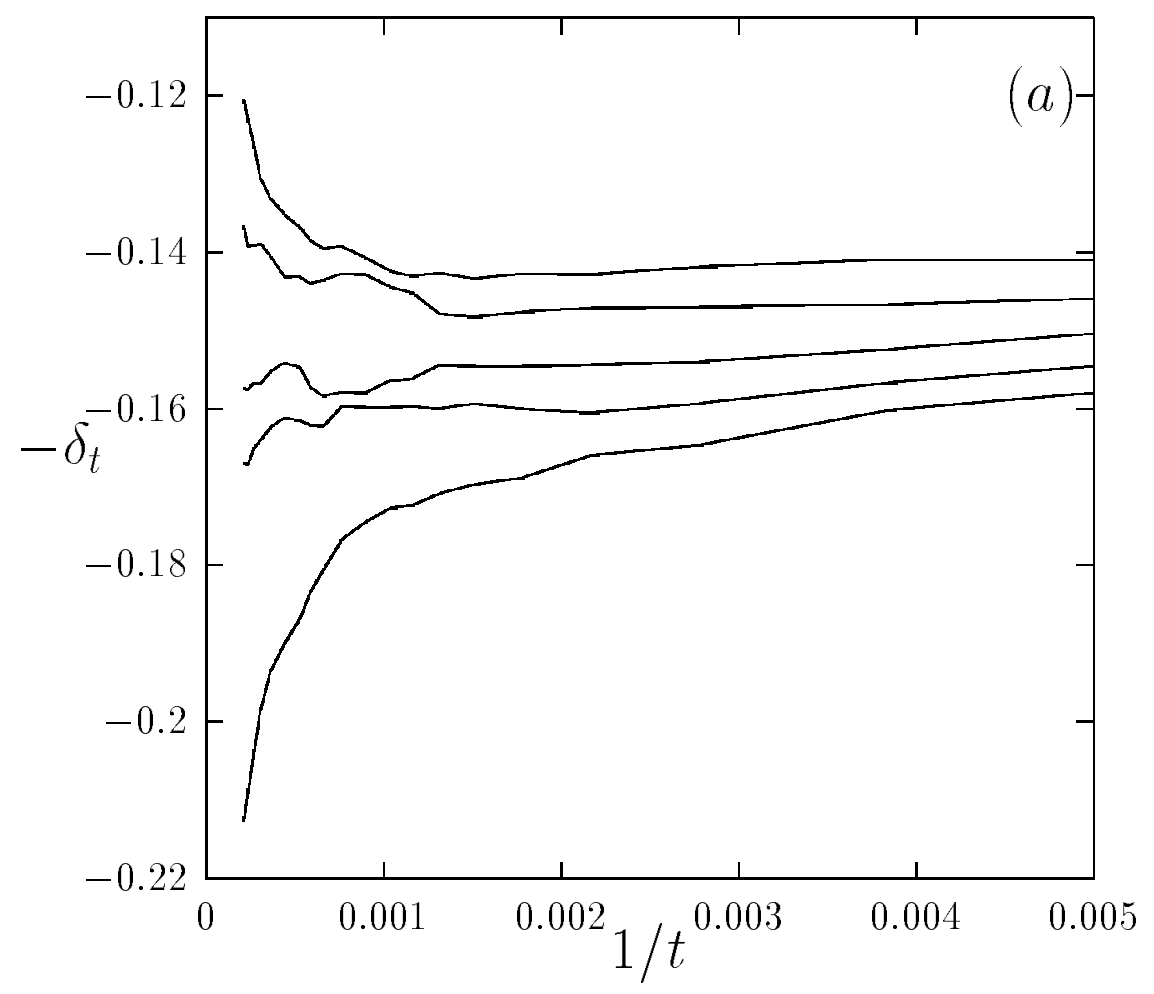

Figure 3(a) 


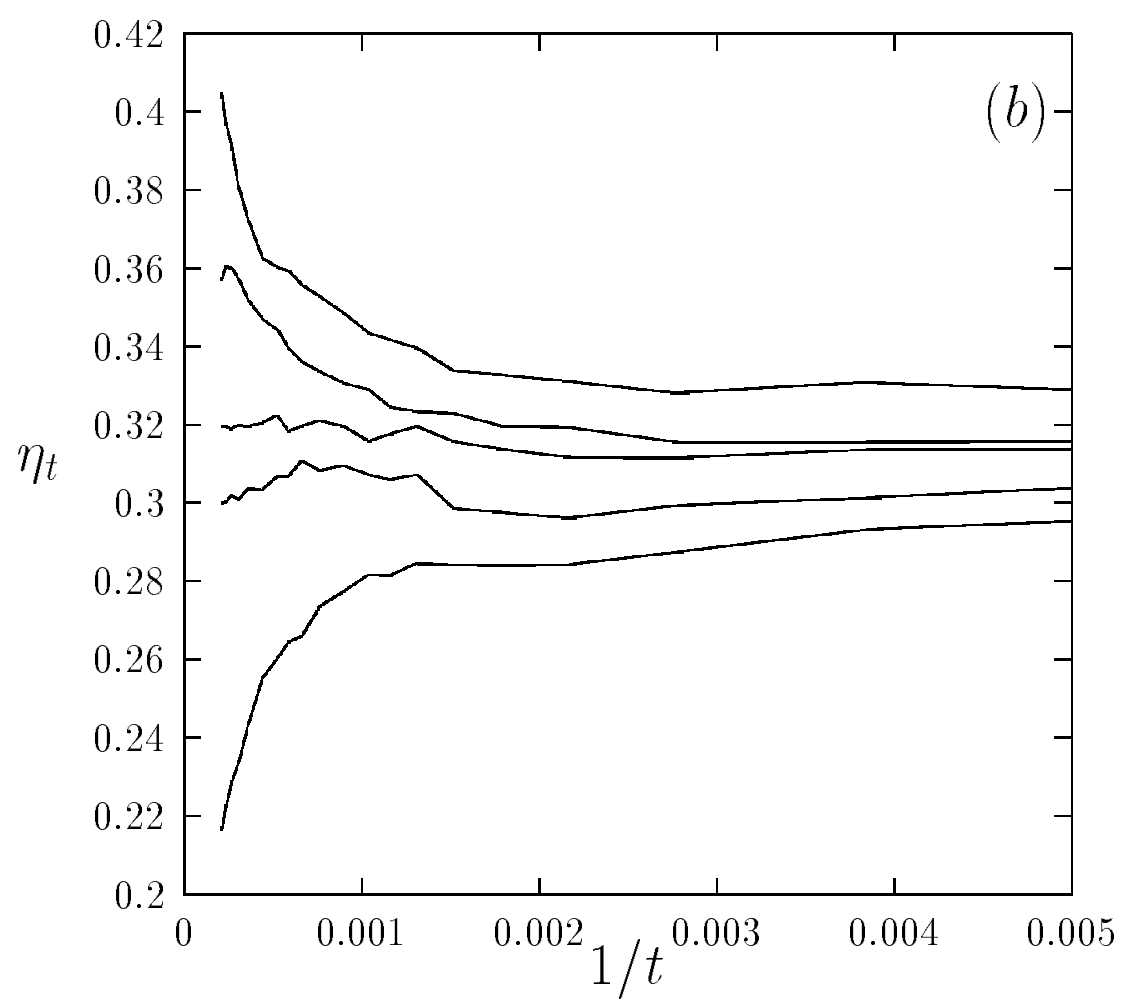

Figure 3(b) 


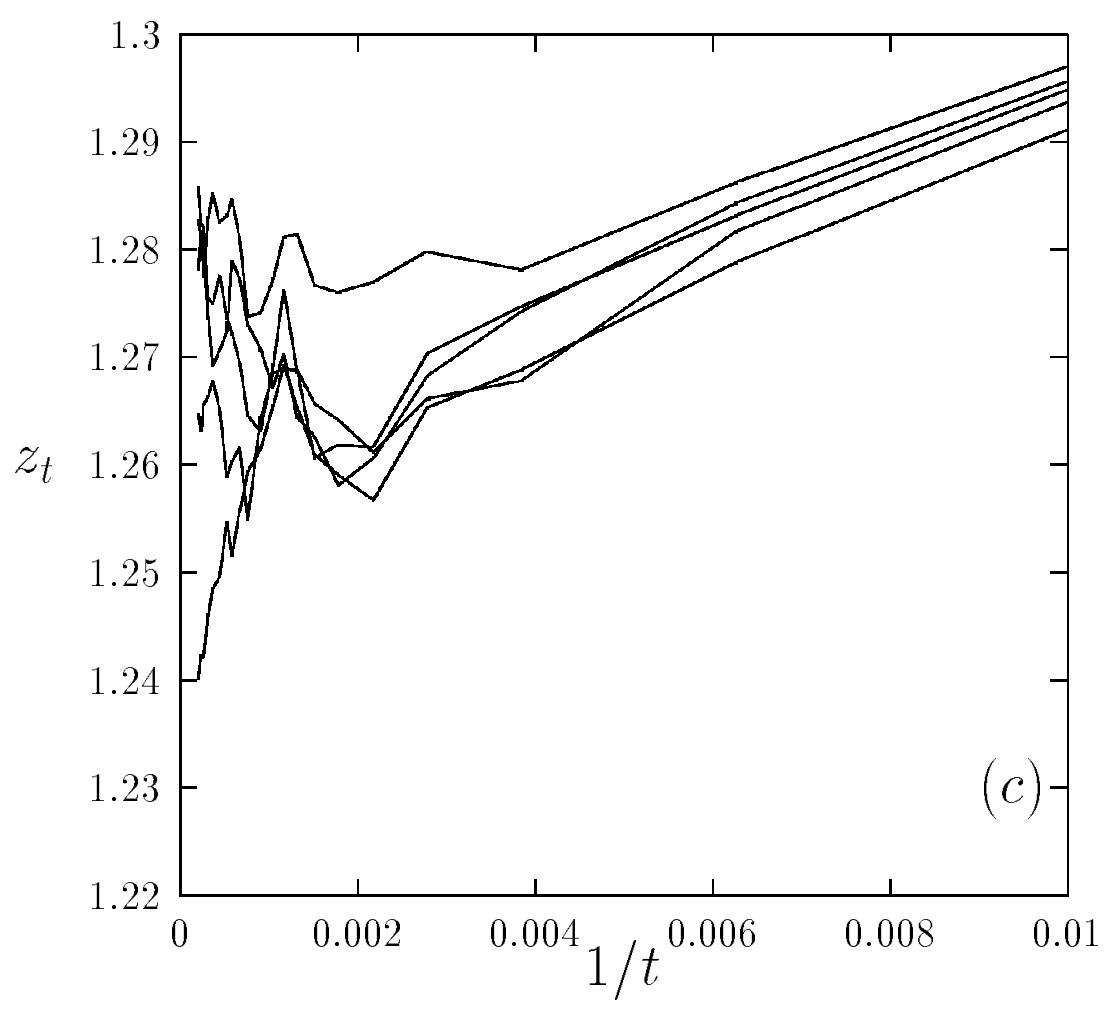

Figure 3(c) 


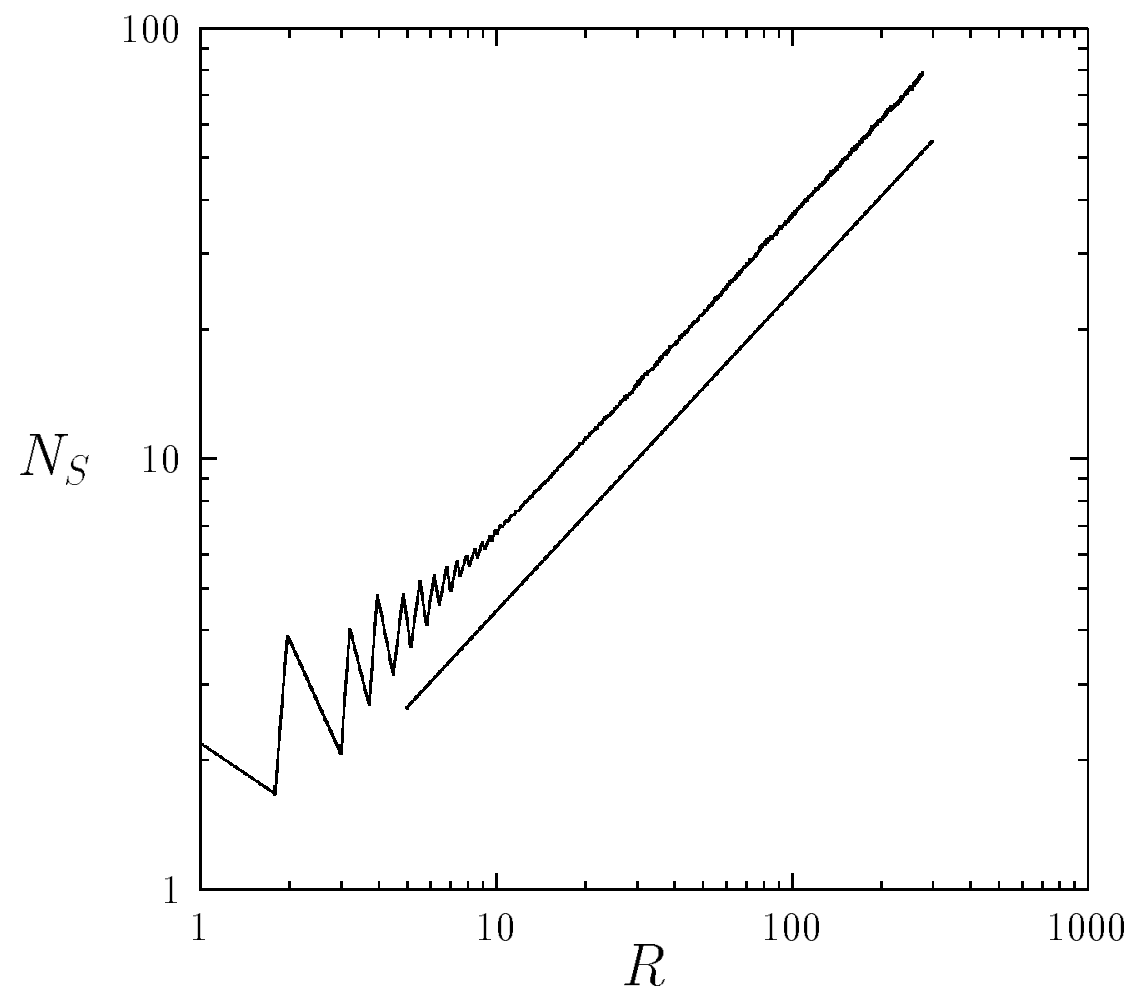

Figure 4 